\title{
Catástrofe de madrugada: rutina periodística, Estado de Excepción y razones de Estado en la información
}

Análisis de las entregas informativas de El Mercurio y La Tercera tras el terremoto del 27 de febrero de 2010 en Chile

\section{Catastrophe of dawn: journalistic routine, state of emergency and reasons of State in the information \\ Analysis of the informative deliveries of El Mercurio and La Tercera after the earthquake of February 27, 2010 in Chile}

\author{
Hernán LÓPEZ GUERRA \\ Universidad de Chile \\ hflopezg@gmail.com
}

Recibido: 22 de junio de 2015 Aceptado y Publicado: 1 de agosto de 2015

\section{Resumen}

Este artículo analiza la contingencia del terremoto en el centro y sur de Chile del 27 de febrero de 2010, a partir de la revisión de la entrega informativa de los diarios El Mercurio y La Tercera. El texto afirma que existen acontecimientos que, al tener características determinadas, se imponen al sistema de comunicación cotidiano e institucionalizado. En este caso, un terremoto. A partir de una investigación analíticadescriptiva, que incluye una extensa revisión metodológica siguiendo una matriz rutino-discursiva elaborada para esta ocasión, se concluye que la rutina periodística, en la caracterización de mundos posibles, establece prácticas discursivas que acuden a la conformación de una contingencia mediática. Así se administra y distribuye la entrega informativa, tomando en cuenta dispositivos y mecanismos como las razones de Estado (estado de excepción, por ejemplo)- que intervienen y complejizan el proceso.

\section{Summary}

The articles analyses the impact of the huge earthquake that took place in Chile in February 27 th of 2010 upon journalistic routines. It is argued that given some specific 
characteristic, events such an earthquake can overcome the institutionalized news media system and its everyday logics. The research relies upon an analyticdescriptive research that includes an extensive methodological observation on the news published in two nationwide newspapers: El Mercurio and La Tercera. It is concluded that journalistic routines, while featuring possible-worlds, set the practices involved in the configuration of a mediated event. Informative outcomes are thus managed and spread by taking into account some intervening elements such as reasons of states or state of emergency which turn the process even more complex.

Palabras claves: Estado de excepción, terremoto, rutina periodística

Key Words: State of exception, earthquake, journalistic routines

\section{Introducción: La Catástrofe de madrugada}

El 27 de febrero de 2010 no hubo certezas. Eran las 03:34:14 de la madrugada, la penúltima madrugada previa al inicio de marzo, la "Catástrofe de madrugada", como la bautizó el diario El Mercurio. Un terremoto de una magnitud de 8,8 grados, con epicentro en el mar frente a las localidades de Curanipe y Cobquecura, despertó a los chilenos entre Valparaíso y Puerto Montt. Allí reside el $80 \%$ de la población del país, con más de 13 millones de personas según la última medición del Instituto Nacional de Estadísticas, en 2002. Los sistemas de comunicación colapsaron, las carreteras se fracturaron y los suministros básicos dejaron de funcionar. Y no fue todo. Minutos más tarde, un tsunami azotó las costas de las localidades previamente devastadas por el sismo. La ineficacia de los mecanismos de emergencia, la descoordinación de las organizaciones de emergencia y la ausencia de información oficial y confiable desde el Gobierno, provocó la inexistencia de una adecuada prevención en la población para enfrentar el maremoto.

Una situación de emergencia, y en particular un desastre natural, devela el rostro sin maquillaje de una nación. En esa línea, los medios de comunicación son, al igual que el Estado, la Iglesia o una empresa, instituciones que también persiguen la normalización de su actividad para precisar la pauta informativa, reordenar la rutina periodística y consolidar la construcción de la nueva e imprevisible contingencia 
mediática. Un ejemplo. El periodista Raúl Fuentes Navarro se encontraba de viaje en Lima cuando, en 1985, un terremoto en México sacudió varias zonas del país. Imposibilitado de regresar debido a los daños, Fuentes Navarro se informa de la contingencia mediática a través de los medios peruanos, a los cuales después de un tiempo analiza. En particular, el periodista trabaja en la construcción del acontecimiento a partir de las fuentes disponibles para una situación de esta naturaleza. Dice Fuentes Navarro (1986):

En el proceso de producción de representaciones sociales del acontecer público seleccionado, los medios recurren a sus fuentes, en este caso agencias transnacionales y televisión pero también boletines institucionales, entrevistas, etc., para constituir sus "objetos de referencia”, es decir, deciden qué dirán sobre el terremoto, lo cual está determinado por los datos de los que disponen y por los principios valorales que sustentan. La escasez de datos no fue obstáculo para caracterizar el terremoto como una catástrofe trágica adjetivada de diversas maneras, pero claramente evaluada (p.40).

Es probable que aquella cobertura, en nuestros días, se hubiese visto fuertemente optimizada. No obstante, el eje central que destaca Fuentes Navarro, y que devela los ejes fundacionales de esta investigación, es fijar la mirada en los actores que intervienen en la construcción del acontecimiento. Los objetos de referencia se constituyen a partir del trabajo periodístico y las fuentes que intervienen. En nuestro caso, la "Catástrofe de madrugada" nos permite pesquisar hasta dónde los campos del Estado y de las instituciones de la sociedad participan y configuran -directa o indirectamente- la nueva contingencia mediática, y de qué manera se articula la conformación de una normalización, o más bien, la consolidación de los mundos posibles. Es claro que hay acontecimientos que se imponen al propio sistema de comunicación institucionalizada al tener unas características determinadas y es evidente que el ejercicio de los medios radica en una sola noticia, que reconfigura la agenda (Rodrigo Alsina, 1993). Sin embargo, es pertinente preguntarnos ¿cuál es la 
rutina periodística que allí se utiliza? ¿Cuáles son los dispositivos que intervienen? ¿De qué forma lo rutinario constituye las prácticas discursivas? Nos importa describir que fue lo que se escribió; también nos importa cómo.

El 28 de febrero, proliferan las escenas de saqueos y pillaje en supermercados y tiendas comerciales de las zonas devastadas por el terremoto. El sociólogo Enrique Gil Calvo (2003) explica que, si bien en una catástrofe la inseguridad en una comunidad aumenta, esto no es sólo porque la inseguridad social genera mayores niveles de riesgo, sino también porque los medios informativos transmiten mayores niveles de alarma social. El Gobierno central decreta zonas de catástrofe a las regiones del Maule y Biobío, y horas más tarde, establece el Estado de excepción, con el consiguiente despliegue de la fuerza militar, la supresión de la libertad de reunión y el toque de queda, entre otros dispositivos.

\subsection{El Gobierno sale en busca del regreso de la normalidad}

En ese escenario, los diarios El Mercurio y La Tercera -medios escritos de mayor circulación a nivel nacional- protagonistas de esta investigación, reproducen la contingencia mediática conteniendo mayores elementos respecto a otros soportes para construir la contingencia mediática. Así, el 01 de marzo de 2010, El Mercurio titula "Gobierno decreta estado de catástrofe y pone militares en la calle para frenar saqueos". La portada es acompañada por una foto de tres militares con ametralladoras, que en su lectura indica que resguardan el orden en Talcahuano. El diario La Tercera realiza un ejercicio similar. La prensa, antes en penumbras, ahora se desenvuelve en un nuevo marco donde esos dispositivos pasan a formar parte de la construcción del acontecimiento. Bien sabemos que el medio de comunicación se consolida en los mismos valores que fundan al Estado liberal democrático, como la supremacía del individuo, el progreso, la verdad y, en último término, la soberanía de la voluntad popular. Así es como la prensa interviene en la deliberación social promoviéndola o restringiéndola, ampliándola u homogeneizándola (Sunkel, 2003). Lo último, considerando a cada una de las empresas informativas desde su 
cosmovisión de mundo, país y sociedad. Así lo entienden los investigadores de la Universidad de Chile Hans Stange y Claudio Salinas (2009):

El periodismo se termina de aprender en el ambiente de trabajo de los propios medios de comunicación. Cada medio posee su ethos (una filosofía, una línea editorial, un estilo de conducción) que establece lo que es periodísticamente posible y recomendable (p.21).

En el caso de El Mercurio y La Tercera, protagonistas del duopolio de la prensa escrita en Chile ${ }^{1}$, existe una cosmovisión de mundo que marca a fuego el editorial y el trabajo periodístico en cada una de sus secciones. ¿Por qué ambos medios? La preeminencia de El Mercurio y La Tercera en el transcurrir de la cotidianidad, no está solo en la construcción de aquellos acontecimientos que marcan una vida, sino además en la incorporación de elementos que aportan a delinear, junto a los campos del Estado y sus contingencias, aquellos caminos que configuran las estructuras de una sociedad. Son los mundos posibles. No solo difunden las ideologías dominantes en democracia liberal moderna; son "organizadores, distribuidores y modeladores de ciertos consensos e interpretaciones de las escenas de la vida diaria" (Salinas, 2007). En una situación de emergencia, entre las pocas certezas por la irrupción de un acontecimiento, esa escena se exacerba. Aún más, la relación entre los campos del Estado y los medios de comunicación, en esa búsqueda de las señales de normalidad, se constituye y fortalece.

Se trata entonces de advertir esa relación entre ambos medios de prensa y los campos del Estado a través de la rutina periodística y las disposiciones del Gobierno central. Es hacer un primer intento de conjugar la ruptura de la cotidianidad y los dispositivos de control, aquellas señales de normalidad que configuran la

\footnotetext{
${ }^{1}$ Durante la dictadura militar, las autoridades clausuraron todos los medios de prensa, salvo aquellos que pertenecían a las empresas El Mercurio y Copesa (que detenta el control de La Tercera). "Una peculiaridad del caso chileno es el rol constituyente de los mercados de comunicación que ha jugado el Estado. Al decir 'constituyente' no nos referimos al rol regulatorio del Estado (...) sino a un rol de moldeamiento del mercado a través de medidas administrativas o políticas" (Sunkel y Geoffroy, 2001, p.115).
} 
construcción del acontecimiento en emergencia -la "Catástrofe de madrugada"- que lo normalizan y delinean el consenso de lo posible.

\section{Metodología: Matriz rutino-discursiva y criterios de selección}

Para operacionalizar los conceptos teóricos que hemos analizado hasta aquí, combinándolos con elementos de análisis de discurso, aplicamos una matriz rutinodiscursiva. La idea es develar las estructuras discursivas, los trazos de la rutina periodística y las razones de Estado en la información. La preeminencia de El Mercurio y La Tercera en el transcurrir de la cotidianidad, no está solo en la construcción de aquellos acontecimientos que marcan una vida, sino además en la incorporación de elementos que aportan a delinear, junto a los campos del Estado y sus contingencias, aquellos caminos que configuran las estructuras de una sociedad. Son los mundos posibles. No solo difunden las ideologías dominantes en democracia liberal moderna; son organizadores, distribuidores y modeladores de ciertos consensos e interpretaciones de las escenas de la vida diaria (Salinas, 2008) junto con esas unidades, se configuran también las exclusiones, la inacción y una cierta conducta de los individuos.

Uno de los autores elegidos es el profesor Claudio Salinas (2008), de la Universidad de Chile, y algunos de los elementos con los cuales construyó una matriz como un instrumento de registro, análisis e interpretación de los editoriales, vinculados directamente a la delincuencia y el delito". El influjo del académico está en consolidar una serie de operaciones para analizar la rutina periodística de ambos medios, a partir de los conceptos enunciados y profundizados en el marco teórico. El segundo nombre al cual hemos acudido para construir la matriz rutino-discursiva es la investigadora austriaca Ruth Wodak (2003) y la inclusión de algunos de los conceptos de análisis del discurso. Y es que la profesora entiende que el conocimiento es la base de la acción formativa que configura la realidad. Explica Wodak: 
Surge la oportunidad no sólo de analizar las prácticas discursivas, sino de analizar también las prácticas no discursivas y las denominadas manifestaciones y materializaciones, así como las relaciones que existen entre esos elementos. Al igual que Foucault, llamo dispositivo a la interacción entre esos elementos (p.69).

De esta manera configuramos el análisis de fragmentos discursivos que componen y construyen el discurso mediático. ¿Cuál es la rutina periodística en emergencia? ¿De qué forma se articula el discurso con las razones de Estado? ¿Cuál es la trayectoria que siguen los editoriales de ambos medios? Para pesquisar estas operaciones hemos fijado criterios de medición que nos permitan analizar las distintas texturas de la contingencia mediática del terremoto y su recorrido desde la irrupción de la "Catástrofe de madrugada" hasta la normalización de la agenda normativa. La idea es denotar los distintos momentos que allí transcurren.

En ese sentido, el primer momento de análisis y revisión comprende desde el día después de ocurrido el terremoto en Chile, primera edición de El Mercurio y La Tercera dedicada al sismo (28 de febrero) y el cambio de mando con la asunción de Sebastián Piñera como Presidente $(11 \text { de marzo })^{2}$. El segundo momento está comprendido entre el 12 de marzo y el 31 de marzo, período en donde la contingencia mediática se concentra en la estructuración del plan de emergencia y reconstrucción y la judicialización -o función punitiva- tras los saqueos y pillaje en algunas zonas devastadas, entre otros. El tercer momento, que comprende todo el mes de abril, establece el proceso de declive final del terremoto como núcleo de la entrega informativa en ambos diarios. Nuevas contingencias mediáticas desplazan

\footnotetext{
${ }^{2}$ El 17 de enero de 2010, el candidato presidencial de la centroderecha, Sebastián Piñera Echeñique, derrotó en un apretado balotaje al abanderado de la centroizquierda, Eduardo Frei Ruiz-Tagle, perteneciente a la Concertación, coalición que gobernó el país durante los últimos 20 años post dictadura militar. Esto último no es un traspaso menor, especialmente considerando que aquellos adherentes políticos al régimen de Augusto Pinochet, en diferentes campos, esta vez por la vía democrática, la oportunidad de regresar al palacio presidencial.
} 
al terremoto, la normalización no sólo indica el reestablecimiento del orden, sino además cambian los énfasis de las prácticas discursivas.

En el primer momento de medición, El Mercurio y La Tercera concentran el despliegue en la contingencia mediática del terremoto. Por lo tanto, y si bien la revisión en los tres momentos tiene como núcleo central los cuerpos nacionales de ambos diarios, entre el 28 de febrero y el 11 de marzo la revisión incluye las secciones Nacional, Internacional y Economía y Negocios. En los siguientes dos momentos temporales, la centralidad está dispuesta en las secciones nacionales. Lo anterior sin perder de vista el seguimiento de algunos temas que aparecen, en algunas ocasiones, en la sección económica (por ejemplo la discusión respecto a una reforma tributaria o el aumento del royalty minero). Además, y durante los dos meses de medición, también se analizan los editoriales de ambos diarios dedicados a la contingencia mediática del terremoto en cualquiera de sus niveles (reconstrucción, saqueos, Onemi, responsabilidades políticas) que nos sirvan para entregar luces respecto a la trayectoria y articulación de la opinión con respecto al acontecimiento.

\begin{tabular}{|c|c|c|c|c|}
\hline $\begin{array}{c}\text { Día } \\
\text { Sección }\end{array}$ & Título & Descripción & Género & $\begin{array}{l}\text { Razones de } \\
\text { Estado en la } \\
\text { información }\end{array}$ \\
\hline & & & $\begin{array}{l}\text { i. Informativo } \\
\text { ii. Descriptivo } \\
\text { iii. Opinión }\end{array}$ & \multirow{3}{*}{$\begin{array}{l}\text { i. Político- } \\
\text { legislativo } \\
\text { ii. Delictual- } \\
\text { punitivo }\end{array}$} \\
\hline & $\begin{array}{l}\text { Lenguaje } \\
\text { utilizado }\end{array}$ & $\begin{array}{l}\text { Construcción de } \\
\text { la información }\end{array}$ & $\begin{array}{l}\text { Fragmentos } \\
\text { discursivos }\end{array}$ & \\
\hline & $\begin{array}{l}\text { i. Citas del } \\
\text { texto }\end{array}$ & $\begin{array}{l}\text { i. Actores } \\
\text { ii. Fuentes } \\
\text { iii. Elementos } \\
\text { adicionales }\end{array}$ & $\begin{array}{l}\text { i. Arquitectura de la } \\
\text { ciudad } \\
\text { ii. Espacio normativo } \\
\text { iii. Proyección de } \\
\text { país }\end{array}$ & \\
\hline
\end{tabular}




\section{Discusión: Construir el acontecimiento}

Tras la irrupción de la contingencia del terremoto, el medio de prensa está obligado a reorganizar su rutina periodística en términos orgánicos. Lo anterior como premisa para consolidar objetos de referencia desde los cuales se articula y caracteriza el acontecimiento; donde se forma conocimiento social. Así lo explica el semiólogo Eliseo Verón (1983):

La opinión (pública) espera del discurso informativo que la mantenga al día sobre lo que ocurre en el mundo. Esta evidencia contiene una serie de presupuestos que intervienen sobre todo en la forma de construir la relación entre el expositor y su destinatario: se admite, por ejemplo, que aquel está en condiciones de determinar la importancia del acontecimiento y que a partir de ahí tendrá que describirlo y explicarlo (p.115).

Entonces, la noticia es obra de un proceso de producción y no sólo un formato de su consecuencia, pues en ella están cifrados los patrones y pautas culturales que se implican en cada una de las prácticas y actores sociales que forman parte de la producción noticiosa. La rutina periodística, entonces, dista de la idea del mediador o de aquel testigo privilegiado. Para Salinas y Stange (2009), la rutina es mucho más que la operación de informaciones, sino más bien la administración de las mismas. Esta lógica también administra "la disposición de los públicos respecto a su realidad social, así como la disposición de los periodistas a su propio proceso y tiempo de producción" (p.24). El periodismo y su práctica no sólo se aprenden en las universidades. Cada medio de comunicación significa ingresar a un ambiente diferente, determinado por las estructuras, ideologías y cultura profesional que ahí se ponen en juego para consolidar una contingencia mediática.

En situación de catástrofe, las estructuras de poder se ven alteradas. En ese contexto, las fuentes del gobierno "emergen como fuentes información de primer 
orden" (Bernardo \& Pelliser, p.108), quienes no solo detentan del carácter oficial, sino además llevan a cuestas la necesidad de entregar señales de normalidad a la población. Para Rodrigo Alsina (1993), esta predominancia sin duda "determina de alguna manera el carácter político de cierta información" (p.95). Es decir, existe una articulación de criterios y valores que intervienen en la producción de la noticia. Más aún, en este caso el medio de prensa -independiente de la orientación que mantenga actual o históricamente- puede transformarse en la reafirmación implícita de los mecanismos o dispositivos del Gobierno como un aparato ideológico. Tras la constatación, horas después de ocurrido el terremoto en Chile, de la fragilidad de los sistemas de comunicación del aparato estatal, el rol de los medios de comunicación y su capacidad de enviar señales de normalidad a la sociedad se vuelve vital. Aún cuando, al igual que las entidades del Estado, las empresas informativas también deben reconstruirse. Las operaciones que se incorporan en la rutina en emergencia se vuelven esenciales, por eso surge una interrogante: ¿cuáles son los dispositivos que intervienen en la construcción del acontecimiento?

\section{Medios y dispositivos}

Para Michelle Foucault (2001), dispositivo es "una especie de formación que tuvo por función mayor responder a una emergencia en un determinado momento. El dispositivo tiene pues una función estratégica dominante" (p.298). A partir de esta definición, el italiano Giorgio Agamben (2005) en una conferencia en Argentina complejiza y amplía el concepto, y se refiere a él como cualquier cosa que tenga de algún modo la capacidad de "capturar, orientar, determinar, interceptar, modelar, controlar y asegurar los gestos, las conductas, las opiniones y los discursos de los seres vivientes". No se trata, entonces, de un término en particular que se refiera solamente a una tecnología del poder, ni tampoco es un mecanismo creado o dado de una casualidad. Cada dispositivo entra en juego para consolidar una red de poder. El dispositivo siempre tiene una función estratégica concreta y se inscribe en una relación de poder. 
Bajo esos márgenes se diseña la sociedad de lo posible, la que Alain Brossat (2008) califica como una "masa previsible, reglamentada, vigilada en todos los sentidos del término" (p.37) y que tiene entre sus dispositivos de control más potentes a los medios de comunicación, que transforman a los individuos como "una masa atomizada, programada y cronometrada" (p.38). Cornelius Castoriadis (2009) afirma que este grupo de dispositivos -entidades fundantes de la sociedad de controlparticipan en la fabricación social del individuo que impregnan al individuo de lo social (p.90). Se trata de "la 'parte' de todas las instituciones que tienden a la educación, al pupilaje, a la educación a los recién nacidos, a lo que los griegos llaman paideia: familia, ritos, escuela, costumbre y leyes, etc." (p.90).

Así, los medios de comunicación, al participar en la articulación de la contingencia mediática, difunden también aquellas ideologías afines, un sistema de representaciones que legitima un sistema político. Es la conformación de los mundos posibles. Sin embargo, esas representaciones entendidas no tan sólo como simple deformadoras de sentido, sino también como posibilitadoras de una ideología. Stuart Hall (2010) entiende que la preeminencia de una determinada ideología en una comunidad tiene sentido sólo en la alianza coyuntural particular con las agencias de las superestructuras, como la Iglesia, la policía o los medios de comunicación. Describe Stuart Hall:

Después de todo, en sociedades democráticas no es una ilusión de la libertad decir que no podemos explicar adecuadamente las inclinaciones estructuradas de los medios como si fueran instruidos por el estado sobre qué deben imprimir o permitir en la televisión. Pero entonces, ¿cómo es que un número tan grande de periodistas, guiándose sólo por su "libertad" para publicar y sufrir las consecuencias, sí tienden a reproducir, espontáneamente, sin obligación, una y otra vez, las visiones del mundo construidas dentro de las mismas categorías ideológicas fundamentales? (p.204). 
Incluso, el sociólogo jamaiquino llega a suponer que, finalmente, hasta los periodistas sensacionalistas terminan inscritos en una ideología que no comparten, pero que "los escribe" (p.205). Esto, justo en el momento en el que hay una ruptura en el orden natural de la dominación y la repartición de roles que determinan la cotidianidad de una sociedad.

\section{Excepcionalidad y Estado}

28 de febrero. Los medios de comunicación, en el marco de la "Catástrofe de madrugada", informan las escenas de saqueos y pillaje en diversos centros comerciales. Lo anterior, especialmente en las zonas con mayor devastación por el terremoto: las regiones del Maule y Biobío. Aparece la violencia como salida de un grupo de individuos a lo vergonzante, a la irrupción de la precariedad. Es también el rechazo a un mundo concebido sin ellos, que se expresa en la devastación de lo propio. Una nueva contingencia que aparece, es la parte que se hace ver. Es el inicio de la política que, según Jacques Rancière (2007), parte con la aparición de la colección de los no contados en el seno de la comunidad, la ruptura de las competencias e incompetencias. "La subjetividad no es ni el trabajo ni la miseria, sino la mera cuenta de los incontados, la diferencia entre la distribución desigualitaria de los cuerpos sociales y la igualdad de los seres parlantes" (p.55).

El quiebre se devela. La "ingobernabilidad" en algunas zonas del país es razón suficiente para que el Gobierno eche mano a los dispositivos de la excepcionalidad: toque de queda, control militar y estado de sitio, entre otros. La señal es enviada: en el Maule y el Biobío entra en funcionamiento el Estado de Excepción, en busca del reorden jurídico y político. Recurrimos a Giorgio Agamben (2003) para definir Estado de Excepción:

El Estado de Excepción se presenta como la apertura en el ordenamiento de alguna laguna ficticia con el objetivo de salvaguardar la existencia de la norma y su aplicabilidad a la situación normal. La laguna no es interna a la 
ley, sino que tiene que ver con su relación con la realidad, la posibilidad misma de su aplicabilidad. Es como si el derecho contuviese una fractura esencial que se sitúa entre la posición de la norma y su aplicación y que, en el caso extremo, puede ser colmada solamente a través del Estado de Excepción, esto es, creando una zona en la cual la aplicación está suspendida, pero la ley permanece, como tal, en vigor (p.70).

Es ese momento en el cual se suspende el derecho precisamente para garantizar su continuidad, su existencia. Es una zona de anomia, y por lo mismo, un lugar en donde lo público y lo privado es desactivado ${ }^{3}$. Los dispositivos del Estado de Excepción entran en acción. Los militares toman el control en busca del orden, la supresión de los saqueos y la atomización de los individuos. En una democracia liberal moderna el Estado, según explica Zygmunt Bauman, "no está dispuesto a quedarse observando con pasividad mientras sus sujetos toman la ley por sus propias manos" (p.100). Es un riesgo demasiado alto que nadie está dispuesto a tolerar. Por ello, aquella ruptura de las relaciones de poder se recompone. Entran en función los dispositivos de la excepcionalidad en la restitución de los mundos posibles, en donde participan los medios de comunicación. Es el río de la biopolítica.

Aquellos individuos que se organizaron no por un "más", sino por un "menos", por una carencia versus aquel que está "exento o eximido" (Esposito, 2003, p.61) deberán volver a ser los incontados. "Es la nuda vida a la que el hombre ha sido reducido, no exige nada ni se adecua a nada: es ella misma la única norma" (Esposito, 2003, p.30). Así es como, finalmente, en palabras de Entel (2007) la eficacia de la ideología "consiste en operar en lo capilar y en lo profundo, de modo

\footnotetext{
${ }^{3}$ En la Constitución de Chile, el Estado de Excepción constitucional puede ser invocado en cuatro "situaciones de excepción": el estado de asamblea, el estado de sitio, el estado de emergencia y el estado de catástrofe. En el caso del estado de catástrofe -declarado en las regiones del Maule y Biobíocarece de un plazo definido. En cada "situación de excepción", el Estado de Excepción limita derechos de acuerdo a la naturaleza del caso. En el estado de catástrofe restringe la circulación de las personas y el transporte de mercaderías; las libertades de trabajo, de información, de opinión y de reunión; dispone requisiciones de bienes y establecer limitaciones al ejercicio del derecho de propiedad; y permite adoptar todas las medidas extraordinarias de carácter administrativo que se estimen necesarias. (Ríos Álvarez, 2002).
} 
de traducirse en comportamientos, en actitudes de aceptación y en otras que demarcan los abyectos a quienes el orden segrega con la complacencia del resto" (p.43).

\section{Resultados}

Interpelan, administran, elaboran. El Mercurio y La Tercera diseñan un mundo posible en el que el orden y el miedo son fundantes. $Y$ lo son por razones de seguridad, de continuidad, de perseguir una estructura de sociedad fracturada y donde ambos medios forman parte de la clase que domina. Están en el lado del poder. Por ello nunca nos debe parecer extraño que las prácticas periodísticas no solo se analicen desde la perspectiva de la profesión. Explican Stange y Salinas (2008):

Las prácticas periodísticas debieran entenderse no sólo sujetas a unas lógicas y a unas relaciones de fuerzas que ocurren al interior del campo periodístico -y que pueden aprehenderse a través de la noción de habitus como un esquema de estrategias e interpretaciones -sino también como formas de expresión de la sociedad, organizadas según su modo de participar en la racionalidad misma (p.38).

Uno de esos mecanismos de contención expresados a través de los medios de prensa es la violencia. Y más en contexto de emergencia. Debemos aplicar "todo el rigor de la ley" a quienes provocan el desajuste, que no es otra cosa que el deterioro más absoluto de la sociedad construida con tanto esfuerzo y por tantos años. La nación pide a los individuos su comprensión y solidaridad a través de los dispositivos del Estado, y que finalmente promueven, en vez de un aumento de la violencia, una reacción de miedo frente a ella. Los académicos italianos Gianfranco Betteleni y Armando Fumagalli (2001), de acuerdo a las constataciones de investigaciones en diversas partes del mundo, llegan a la siguiente conclusión: 
El aumento del comportamiento agresivo, la disminución de la sensibilidad hacia la violencia real y el aumento injustificado del miedo, pueden ser considerados los principales efectos a largo plazo de la representación de la violencia llevada a cabo por los medios (p.182).

Un ejemplo claro es la contingencia de los saqueos. Muy temprano y con mucha celeridad, una vez ocurridos los hechos en las zonas de emergencia ambos medios demarcan claramente quién es la víctima y quién el victimario. Pero no sólo eso, a través de los editoriales y del enfoque de una buena cantidad de notas, El Mercurio y La Tercera exigen que la función punitiva demuestre a la sociedad en su conjunto cuáles son los márgenes entre los cuales pueden moverse los ciudadanos. Serán los marcos de su libertad. Entonces, diagraman "la aprobación o desaprobación de un comportamiento, el juzgarlo bueno o malo, según los criterios que pueden variar en relación con las motivaciones puestas en evidencia y argumentadas para la narración" (Betteleni \& Fumagalli, p.186). Lo anterior es una trayectoria compartida, una especie de consenso implícito en el cual El Mercurio y La Tercera comparten un mundo posible. La articulación es total, aquella clausura es significado de un Estado que va más allá de lo político o jurídico; es racionalidad. Está inscrito. ¿Quién garantiza la unidad nacional? ¿Quién defiende los intereses de la nación?

Hay algo aún más relevante en la estrategia que utilizan ambos diarios, que esbozamos más atrás y que es reconocible en el editorial: la responsabilidad política versus la épica del terremoto. Esto es central: mientras La Tercera exalta el ideario nacional respecto a la "solidaridad de los chilenos", El Mercurio no pierde tiempo y reclama las responsabilidades políticas por las descoordinaciones en los momentos posteriores al sismo. Sin embargo, ambos coinciden en defender la implementación del Estado de Excepción y sus dispositivos en defensa de la seguridad. Es la cristalización de la racionalidad. La racionalidad pone en marcha lo posible, que no es otra cosa que el retorno de una normalidad que transita en un desajuste. Es restablecer el poder, pues la pérdida de éste significa socavar la comunidad 
armoniosamente constituida. Por ello la apuesta por los dispositivos de la excepcionalidad. Dice Hannah Arendt (1993):

El poder no puede almacenarse y mantenerse en reserva para hacer frente a las emergencias, como los instrumentos de la violencia, sino que sólo existe en su realidad. Donde el poder carece de realidad, se aleja, y la historia está llena de ejemplos que muestran que esta pérdida no pueden compensarla las mayores riquezas materiales (p.223).

Durante marzo, la necesidad de intervención transita hacia la difusión del miedo. El discurso distribuido por ambos medios ha cumplido su cometido, y la judicialización de los saqueos lleva, paulatinamente, a la normalización. La Tercera habla del "regreso a la tranquilidad", mientras que El Mercurio se refiere a la "amenaza de allanamientos" como razón para la devolución de los artículos sustraídos. A través de la práctica discursiva podemos descifrar una rutina que apunta a la construcción de un acontecimiento que promueve la legitimidad de la acción militar, la entrada en vigencia de los dispositivos del Estado de Excepción y la supuesta valoración de la ciudadanía. No perdamos de vista que Carabineros fue la segunda institución mejor evaluada y el Ejército la quinta en la evaluación del Centro de Estudios Públicos tras el sismo. Lo último, a pesar de los desencuentros con el Ejecutivo por la reacción durante las horas posteriores al terremoto. Por ello es que, incluso, tal como informó el diario La Tercera, no solo se da cuenta de un correlato que termina con el traspaso de las fuerzas del Ejército a labores de reconstrucción, sino además participa de ese proceso punitivo de condena a los saqueos facilitando fotografías para determinar a los responsables.

La rutina periodística de un diario no sólo diseña la edición que vendrá. Jerarquiza y dispone de una entrega informativa que vaya de acuerdo con la construcción de ese mundo posible. En el caso de El Mercurio, las operaciones que allí se ejecutan apuntan a consolidar un ideario de país, un modelo de sociedad. No sólo promueve la identificación de los responsables de la tragedia; proyecta una serie de reformas en 
diversos campos del Estado. Un nuevo entramado que no permita los desajustes propios de la desesperación bajo incertidumbre. En tanto, La Tercera apela en un primer momento a la exaltación de lo nacional, lo que días más tarde se va a transformar en la negativa más rotunda a cualquier reforma en el campo económico que se traduzca, por ejemplo, en el aumento de impuestos. ¿Qué queremos decir con esto? Que la expresión de ideologías de ambos medios y su participación en el mundo posible plantea una advertencia: o se profundiza lo que hay (El Mercurio) o se protege lo que existe (La Tercera). Ambos medios son parte de un grupo que no sólo posee intereses particulares, sino también ideas que se expresan y constituyen imágenes de mundo. Un mundo posible que los ubica en el sector dominante.

¿Cuál es la diferencia entre una rutina en emergencia y una rutina "normal"? Ya hemos esbozado un intento de respuesta en capítulos anteriores. Podríamos separar la rutina en dos ámbitos para desarrollar un mejor análisis. Un primer análisis es de tipo "orgánico". Aquí claramente el trabajo del medio, debido a la irrupción, cambia. Todo se centraliza en una sola contingencia, y se dispone de todo el material técnico y humano para desarrollarla. $Y$ es evidente, porque ambos medios buscan liderar la agenda informativa y deben luchar contra otros soportes (como la televisión o la radio) que cuentan con la inmediatez como principal herramienta. Aquella disposición no cambia ni en El Mercurio ni en La Tercera. No obstante, un segundo análisis de carácter más "político" nos indica que la rutina sigue siendo la misma. Una misma reunión de pauta -con los mismos editores que día a día interactúan- dispone la entrega informativa con los énfasis que tradicionalmente utiliza: la formación profesional y el conocimiento social que se articulan con los preceptos del medio en el cual se trabaja. En esa línea, la expresión de violencia y miedo para restablecer el orden fundante no es otra cosa que la expresión de la rutina periodística y los contornos del Gobierno, expresados en las razones de Estado en la información. A través de su entrega, van generalizando y totalizando las interpretaciones respecto a la cotidianidad, lo que configura un escenario de legitimidad del orden actual. El desplazamiento de la emergencia como contingencia de la agenda mediática tiene 
que ver con la normalización de la comunidad. Ahora que el orden ha sido reimpuesto, podemos ocuparnos de otros acontecimientos. La rutina, con algunos ajustes de tipo “orgánico", continúa funcionando sin mayores problemas.

Pero orden y miedo solo son fundantes en términos de hegemonía. Y si bien en esta investigación analizamos el término a partir del control de los aparatos ideológicos del Estado y de la supremacía de una ideología, queremos hacer hincapié en dos conceptos. El primero de ellos promovido por Antonio Gramsci (1981):

En el sistema hegemónico existe democracia entre el grupo dirigente y los grupos dirigidos, en la medida en que el desarrollo de la economía y por consiguiente de la legislación, que expresa tal desarrollo, favorece el pasaje de los grupos dirigidos al grupo dirigente (p.123).

El segundo de ellos, del filósofo y sociólogo Néstor García Canclini (1984), tomando elementos de Gramsci y del sociólogo francés Pierre Bordieu:

La hegemonía es entendida como un proceso de dirección política e ideológica en el que una clase o sector logra una apropiación preferencial de las instancias de poder en alianza con otras clases, admitiendo espacios donde los grupos subalternos desarrollan prácticas independientes y no siempre "funcionales" para la reproducción del sistema (p.72).

¿Por qué utilizamos estos conceptos? Para realizar una proyección. El estudio respecto a rutinas periodísticas no es cuantioso. Esta investigación pretende servir como insumo a ese debate, reconociendo los márgenes acotados del trabajo -el contexto de emergencia tras el terremoto del 27 de febrero en Chile- y con particularidades tan marcadas como la contingencia mediática de los saqueos o el Estado de Excepción. Sin embargo, en esas particularidades también hay problemas que pueden ser abordados como próximas investigaciones. ¿Qué pasa con los incontados? En la hegemonía democrática, tal como hemos reseñado, aún quedan espacios para desarrollar "prácticas independientes" y "no siempre funcionales" al 
sistema actual. ¿Cuáles serían ésas, por ejemplo, en un contexto de emergencia? ¿Obligaría a un rediseño de la rutina periodística? ¿Podría existir una relación entre el discurso mediático y el "pasaje" de los grupos dirigidos al grupo dirigente? Son interrogantes interesantes para profundizar, porque permiten establecer un relato, una continuidad respecto al rol de los medios de comunicación. También es bueno dejar en claro que las particularidades de una irrupción -como en este caso fue el terremoto- permiten seguir el desarrollo de otros eventos. No es lo mismo una catástrofe natural a un conflicto armado, a una revuelta social o a un atentado terrorista. Son todos escenarios que permiten proyectar nuevas e interesantes líneas de investigación.

\section{Referencias bibliográficas}

Agamben, G. (2003). Estado de excepción. Adriana Hidalgo Editora, Buenos Aires.

Agambem, G. (2005). ¿Qué es un dispositivo? Conferencia en la Universidad Nacional de La Plata. http://ayp.unia.es/r08/IMG/pdf/agamben-dispositivo.pdf

Arendt, H. (1993). La condición humana. Editorial Paidós, Madrid, España.

Bauman, Z. (2004). La sociedad sitiada. Fondo de cultura económica, Ciudad de México, 2004.

Bernardo, J. \& Pelliser, N. (2010). La naturalización mediática de las catástrofes. Una aproximación crítica. En: cuadernos de información facultad de comunicaciones Universidad Católica, Santiago de Chile.

Betteleni, G. \& Fumagalli, A. (2001). Lo que queda de los medios. Ediciones Universidad de Navarra, Navarra.

Brossat, A. (2008). La democracia inmunitaria. Editorial Palinodia, Santiago.

Castoriadis, C. (2009). El mundo fragmentado. Editorial Terramar, Buenos Aires.

Entel, A. (2007). La ciudad y los miedos. Editorial La Crujía, Buenos Aires.

Esposito, R. (2003). Conmunitas. Editorial Amorrortu, Buenos Aires. 
Foucault, M. (2001). Dits et ecrtis III. Gallimard, Paris.

Fuentes Navarro, R. (1986). La construcción informativa del acontecer. El terremoto de México en los diarios de Lima. Editorial Iteso, Ciudad de México.

García Canclini, N. (1984). Gramsci con Bourdieu. Hegemonía, y nuevas formas de organización popular. En: revista Nueva Sociedad, n71.

Gil Calvo, E. (2003). El miedo es el mensaje: riesgo, incertidumbre y medios de comunicación. Alianza Editorial, Madrid.

Gramsci, A. (1981). La política y el Estado moderno. Premia Editora, México.

Hall, S. (1996). Cuestiones de identidad cultural. Amorrortu Editores, Madrid.

Rancière, J. (2008). El espectador emancipado. Editorial Manantial, Buenos Aires.

Rodrigo Alsina, M. (1993). La construcción de la noticia. Editorial Paidós, Barcelona.

Salinas, C. (2007) El Mercurio y Plan Z: El periodismo no ha tenido lugar. Memoria para optar al título de periodista Universidad de Chile, Santiago

Salinas, C. (2008). La seguridad y la administración mediática del miedo. El delito en las editoriales de El Mercurio y La Tercera. Tesis de Magíster en Comunicación Política Universidad de Chile, Santiago.

Stange, H. \& Salinas, C. (2009). Rutinas periodísticas, discursos y trayectos teóricos sobre el concepto y estudio de la prensa chilena. Cuadernos Icei, Santiago.

Sunkel, G. \& otros (2003). Participación y democracia en la sociedad de la información. Editorial Prometeo, Buenos Aires.

Verón, E. (1983). Construir el acontecimiento. Los medios de comunicación masiva y el accidente en la central nuclear de Three mile Island. Editorial Gedisa. Buenos Aires.

Wodak, R. (2003). Métodos de análisis crítico del discurso. Editorial Gedisa, Madrid.

\section{Forma de citar este artículo en bibliografías}

LÓPEZ GUERRA, Hernán (2015): "Catástrofe de madrugada: rutina periodística, Estado de Excepción y razones de Estado en la información. Análisis de las entregas informativas de El Mercurio y La Tercera tras el terremoto del 27 de febrero de 2010 en Chile", en Revista PANGEA, 6, páginas 136 a 155. Red Académica Iberoamericana de Comunicación. Recuperado el de de 2 de: http://www.revistapangea.org 